\title{
Detection and Isolation of Actuator/Surface Faults for a Large Transport Aircraft
}

\author{
Andras Varga
}

\begin{abstract}
We consider the problem of designing residual generators with least dynamical orders to solve actuator fault detection and isolation problems for a Boeing $747-100 / 200$ aircraft. The main result of our analysis is the proof of feasibility of the complete isolation of all primary actuator/surface faults in the nominal case by using a minimal number of additional surface angle sensors. The analysis of the nominal case provides residual filter specifications which can be employed in a more realistic design, where robustness aspects with respect to external noise (gusts, measurements) and parametric/flight condition uncertainties are also considered.
\end{abstract}

\section{Introduction}

In this chapter we address the problem of detection and isolation of actuator faults for a Boeing 747-100/200 from the perspective of fault tolerant control (FTC). The main goal of FTC is to allow, after a successful identification of faults, the application of appropriate control reconfiguration to ensure safe operation of the aircraft in the presence of identified failures or, in extreme cases, to guarantee a safe landing to the nearest airport. The most relevant faults for our analysis are related to four categories of primary control surfaces: elevator, stabilizer, rudder, and ailerons.

In numerous studies, the occurrence of actuator faults for the Boeing 747$100 / 200$ aircraft has been addressed in a simplistic way, by assuming that all faults related to a surface category occur simultaneously [1, 2]. For example, it is usually assumed that all four elevators are simultaneously affected by the same fault or, equivalently, each elevator fault is assimilated with a global fault on all elevator surfaces. As a consequence, the typical approach to compensate for elevator faults is to use the stabilizer for the aircraft altitude control and ignore the possibility of em-

German Aerospace Center, DLR - Oberpfaffenhofen

Institute of Robotics and Mechatronics

D-82234 Wessling, Germany. e-mail: Andras.Varga@dlr.de 
ploying, for the same purpose, the remaining healthy elevator surfaces. For the purpose of FTC, such a simplifying assumption of simultaneous elevator faults prevents exploiting the existing freedom in using healthy surfaces which could compensate (fully or partially) the disturbance induced by the faulty surfaces.

This way of addressing the fault occurrence aspect is clearly not appropriate for the purpose of FTC, where precise information on the available healthy actuators/surfaces and faulty ones could be vital for an appropriate control reconfiguration. The existing redundancy in the control surfaces makes it easier to cope with partial failures providing an increased overall safety. Thus, handling only complete surface failures is not a realistic option for FTC.

In this paper we focus on the design of residual generators with least dynamical orders to solve actuator fault detection and isolation problems for the Boeing $747-100 / 200$ aircraft. The main result of our analysis is the proof of feasibility of the complete isolation of all primary actuator/surface faults in the nominal case by using a minimal number of additional surface angle sensors. The analysis of the nominal case provides residual filter specifications which can be employed in a more realistic design, where robustness aspects with respect to external noise (gusts, measurements) and parametric/flight condition uncertainties are also considered.

The paper is organized as follows. First we briefly review the solution of the fault detection problem using scalar output detectors with least dynamical order. The corresponding design procedure is based on the nullspace method in combination with dynamic cover techniques. This method is the basis for the design of a bank of residual generators to solve the more involved fault detection and isolation problems, where a given fault-to-residual influence structure must be achieved. The design methods of residual generators for fault detection and isolation have been recently implemented as robust numerical software, which extends the Fault Detection Toolbox [3] of DLR. The new tools were used to study the feasibility of complete fault detection and isolation of actuator faults for a Boeing 747-100/200 aircraft. Fault detection both at component (actuator) level as well as at the system level are discussed. Residual synthesis results are presented for detecting and isolating both longitudinal and lateral axis failures for several influence structures of increasing complexity. The main result of out study is the solution of the complete isolation problem by employing a minimum number of additional surface sensors.

\section{Design of Least Order Scalar Output Detectors}

Consider the linear time-invariant system described by the input-output relations

$$
\mathbf{y}(s)=G_{u}(s) \mathbf{u}(s)+G_{d}(s) \mathbf{d}(s)+G_{f}(s) \mathbf{f}(s),
$$

where $\mathbf{y}(s), \mathbf{u}(s), \mathbf{d}(s)$, and $\mathbf{f}(s)$ are Laplace-transformed vectors of the $p$-dimensional system output vector $y(t), m_{u}$-dimensional control input vector $u(t), m_{f^{-}}$ dimensional fault signal vector $f(t)$, and $m_{d}$-dimensional disturbance vector $d(t)$, 
respectively, and where $G_{u}(s), G_{f}(s)$ and $G_{d}(s)$ are the transfer-function matrices (TFMs) from the control inputs to outputs, fault signals to outputs, and disturbances to outputs, respectively.

To detect faults, residual generator filters (or fault detectors) having the general form

$$
\mathbf{r}(s)=R(s)\left[\begin{array}{l}
\mathbf{y}(s) \\
\mathbf{u}(s)
\end{array}\right]
$$

are employed, where $r(t)$ is the residual signal generated from the available measurements $y(t)$ and control inputs $u(t)$. A residual generator must fulfill two basic requirements: 1) to generate zero residuals in the fault-free case, for arbitrary control and disturbance inputs; 2) to generate nonzero residuals when any fault occurs in the system. These requirements can be made precise as follows:

Fault Detection Problem (FDP): Determine a proper and stable linear residual generator having the general form (2) such that:

(i) $r(t)=0$ when $f(t)=0$ for all $u(t)$ and $d(t)$;

(ii) $r(t) \neq 0$ when $f_{i}(t) \neq 0$, for $i=1, \ldots, m_{f}$.

In addition to the above requirements, it is often necessary for practical use that the TFM of the detector $R(s)$ has the least possible McMillan degree. Note that as a fault detector, we can always choose $R(s)$ as a rational row vector.

The fulfillment of requirement $(i i)$ ensures that faults produce non-zero residual responses. When designing fault detectors this requirement for fault detectability is usually replaced by the stronger request that persistent (constant) faults produce asymptotically persistent (constant) residuals. This requirement is known as strong fault detectability and has a special importance for practical applications [22].

Let $G_{f_{i}}(\lambda)$ be the $i$-th column of $G_{f}(\lambda)$. A necessary and sufficient condition for the existence of a solution of the FDP is the following $[4,5]$ :

Theorem 1. For the system (1) the FDP is solvable iff

$$
\operatorname{rank}\left[G_{d}(\lambda) G_{f_{i}}(\lambda)\right]>\operatorname{rank} G_{d}(\lambda), i=1, \ldots, m_{f}
$$

The requirements $(i)$ and $(i i)$ of the FDP can be easily transcribed into equivalent algebraic conditions. Condition $(i)$ is equivalent to

$$
R(s) G(s)=0
$$

where

$$
G(s)=\left[\begin{array}{cc}
G_{u}(s) & G_{d}(s) \\
I_{m_{u}} & 0
\end{array}\right],
$$

while the detectability condition (ii) is equivalent to

$$
R_{f_{i}}(s) \neq 0, \quad i=1, \ldots, m_{f}
$$

where $R_{f_{i}}(s)$ is the $i$-th column of 


$$
R_{f}(s):=R(s)\left[\begin{array}{c}
G_{f}(s) \\
0
\end{array}\right]
$$

Enforcing the strong detectability of constant faults is equivalent to ensuring finite non-zero DC-gains for each column of $R_{f}(s)$, i.e.

$$
0<\left\|R_{f_{i}}(0)\right\|<\infty, \quad i=1, \ldots, m_{f}
$$

Conditions (4) and (6) (or (8)) lead to a straightforward design procedure:

\section{FD Least Order Synthesis Procedure}

1. Compute a minimal basis $N_{l}(s)$ for the left nullspace of $G(s)$.

2. Choose a rational vector $h(s)$ such that

$$
R(s)=h(s) N_{l}(s)
$$

has least McMillan degree and fulfills (6) (or (8)).

3. If necessary, replace $R(s)$ by $m(s) R(s)$, where $m(s)$ is chosen to achieve a desired dynamics for the resulting detector.

The scalar output detector $R(s)$ at Step 2) is determined as a linear combination of the basis vectors (rows of $N_{l}(s)$ ), such that conditions (6) or (8) are fulfilled. The above expression for $R(s)$ represents a parametrization of all possible detectors and is the basis for the class of nullspace methods introduced in [6]. While this work relies on using polynomial nullspace bases for $N_{l}(s)$, an alternative approach relying on proper rational bases has been proposed by the author in [7]. The main advantage of this latter method is to rely exclusively on reliable numerical techniques based on state-space computations (see Section 4).

\section{Solving Fault Isolation Problems}

The more advanced functionality of fault isolation (i.e., obtaining the exact location of faults) can be often achieved by designing a bank of fault detectors [8] or by direct design of fault isolation filters [9]. Designing detectors which are sensitive to some faults and insensitive to others can be reformulated as a standard FDP, by formally redefining the faults to be rejected in the residual as fictitious disturbances.

Let $R(s)$ be a given detector and let $R_{f}(s)$ be the corresponding fault-to-residual TFM in (7). We denote $R_{f_{j}}^{i}(s)$ as the $(i, j)$ entry of $R_{f}(s)$. We define the fault signature matrix $S$, with $(i, j)$ entry $S_{i j}$ given by 


$$
\begin{aligned}
S_{i j}= & 1 \text { if } R_{f_{j}}^{i}(0) \neq 0 \\
S_{i j}=-1 & \text { if } R_{f_{j}}^{i}(0)=0 \text { and } R_{f_{j}}^{i}(s) \neq 0 \\
S_{i j}= & \text { if } R_{f_{j}}^{i}(s)=0
\end{aligned}
$$

If $S_{i j}=1$ then we say that the fault $j$ is strongly detected in residual $i$. If $S_{i j}=-1$ then the fault $j$ is only weakly detected in residual $i$. The fault $j$ is not detected in residual $i$ if $S_{i j}=0$.

The following fault detection and isolation problem (FDIP) can now be formulated: Given a $q \times m_{f}$ fault signature matrix $S$ determine a bank of $q$ stable and proper scalar output residual generator filters

$$
\mathbf{r}_{i}(s)=R^{i}(s)\left[\begin{array}{l}
\mathbf{y}(s) \\
\mathbf{u}(s)
\end{array}\right], i=1, \ldots, q
$$

such that, for all $u(t)$ and $d(t)$ we have:

(i) $r_{i}(t)=0$ when $f_{j}(t)=0, \forall j$ with $S_{i j} \neq 0$;

(ii) $r_{i}(t) \neq 0$ when $f_{j}(t) \neq 0, \forall j$ with $S_{i j} \neq 0$.

In this formulation of the FDIP, each scalar output detector $R^{i}(s)$ achieves an influence structure representing the $i$-th row of the desired fault signature structure matrix $S$. For example, to achieve the complete isolation of a maximum of $k$ simultaneous faults, the choice $S=I_{k}$ is necessary. In many practical applications this strong isolation can not be achieved due to the lack of sufficient number of measurements. If we can assume that the faults occur one at a time, a so-called weak isolation of $k$ faults could be possible by using a fault signature matrix whose $i$-th row contains all ones except the element in column $i$ which is zero. For example, for 3 faults $S$ is chosen as

$$
S=\left[\begin{array}{lll}
0 & 1 & 1 \\
1 & 0 & 1 \\
1 & 1 & 0
\end{array}\right]
$$

If this fault signature specification can be achieved, then the occurrence of fault $i$ can be detected if all residuals (excepting the $i$-th residual) are non-zero. More insight into how to specify fault signature matrices can be found in [10].

Let $S$ be a given $q \times m_{f}$ fault signature matrix and denote by $\bar{G}_{f}^{i}(s)$ the matrix formed from the columns of $G_{f}(s)$ whose column indices $j$ correspond to zero elements in row $i$ of $S$. The solvability conditions of the FDIP build up from the solvability of $q$ individual FDPs.

Theorem 2. For the system (1) the FDIP with given fault signature matrix $S$ is solvable if and only if for each $i=1, \ldots, q$, we have

$$
\operatorname{rank}\left[G_{d}(s) \bar{G}_{f}^{i}(s) G_{f_{j}}(s)\right]>\operatorname{rank}\left[G_{d}(s) \bar{G}_{f}^{i}(s)\right]
$$

for all $j$ such that $S_{i j} \neq 0$.

The standard approach to determine $R(s)$ is to design for each row $i$ of the fault signature matrix $S$, a detector $R^{i}(s)$ which generates the $i$-th residual signal $r_{i}(t)$, 
and thus represents the $i$-th row of $R(s)$. For this purpose, the nullspace method can be applied with $G(s)$ in (5) replaced by

$$
G(s)=\left[\begin{array}{ccc}
G_{u}(s) & G_{d}(s) & \bar{G}_{f}^{i}(s) \\
I_{m_{u}} & 0 & 0
\end{array}\right]
$$

and with a redefined fault to output TFM $\widetilde{G}_{f}^{i}(s)$, formed from the columns of $G_{f}(s)$ whose indices $j$ correspond to $S_{i j} \neq 0$.

The resulting global detector can be assembled as

$$
R(s)=\left[\begin{array}{c}
R^{1}(s) \\
\vdots \\
R^{q}(s)
\end{array}\right]
$$

and has a total McMillan degree which is bounded by the sum of the McMillan degrees of the component detectors. Note that this upper bound can be effectively achieved, for example, by choosing mutually different poles for the individual detectors.

Using the least order design techniques described in this paper, for each row of $S$ we can design a scalar output detector of least McMillan degree. However, even if each detector has the least possible order, there is generally no guarantee that the resulting order of $R(s)$ is also the least possible one. To the best of our knowledge, the determination of a detector of least global McMillan degree for a given fault signature $S$ is still an open problem. A solution to this problem has been recently suggested in [11] and is summarized in the following synthesis procedure:

\section{FDI Synthesis Procedure}

1. For $i=1, \ldots, q$

a. Redefine disturbance vector $d$ to include all faults $f_{j}$ for which $S_{i j}=0$.

b. Redefine fault vector $f$ by deleting all faults $f_{j}$ for which $S_{i j}=0$.

c. Compute $R^{i}(s)$ of order $v_{i}$ using the FD Least Order Synthesis Procedure.

2. Ensure that for $v_{i} \leq v_{j}$, the poles of $R^{i}(s)$ are among the poles of $R^{j}(s)$.

3 . Form the global detector $R(s)$ according to (11).

It was conjectured in [11] that the McMillan degree of $R(s)$ resulting from this procedure is the least possible one.

We describe now an enhanced two step approach to design a bank of detectors, which for larger values of $q$, is potentially more efficient than the above standard approach. In a first step, we can reduce the complexity of the original problem by decoupling the influences of disturbances and control inputs on the residuals. In a 
second stage, a residual generation filter is determined for a system without control and disturbance inputs which achieves the desired fault signature.

Let $N_{l}(s)$ be a minimal left nullspace basis for $G(s)$ defined in (5) and define a new system without control and disturbance inputs as

$$
\widetilde{\mathbf{y}}(s):=N_{f}(s) \mathbf{f}(s),
$$

where

$$
N_{f}(s):=N_{l}(s)\left[\begin{array}{c}
G_{f}(s) \\
0
\end{array}\right]
$$

The system (12) has generally a reduced McMillan degree [12] and also a reduced number of outputs $p-r_{d}$, where $r_{d}$ is the normal rank of $G_{d}(s)$.

For the reduced system (12) with TFM $N_{f}(s)$ we can determine, using the FDI Synthesis Procedure, a bank of $q$ scalar output least order detectors of the form

$$
\mathbf{r}_{i}(s)=\widetilde{R}^{i}(s) \widetilde{\mathbf{y}}(s), i=1, \ldots, q
$$

such that the same conditions are fulfilled as for the original FDIP. The TFM of the final detector can be assembled as

$$
R(s)=\left[\begin{array}{c}
\widetilde{R}^{1}(s) \\
\vdots \\
\widetilde{R}^{q}(s)
\end{array}\right] N_{l}(s)
$$

Comparing (15) and (11) we have

$$
R^{i}(s)=\widetilde{R}^{i}(s) N_{l}(s)
$$

which can be also interpreted as an updating formula of a preliminary (incomplete) design. The resulting order of the $i$-th detector is the same as before, but this two step approach has the advantage that the nullspace computation and the associated least order design involve systems of reduced orders (in the sizes of state, input and output vectors).

The above procedure has been used for the example studied in [13, Table 2], where a $18 \times 9$ fault signature matrix $S$ served as specification. Each line of $S$ can be realized by a detector of order 1 or 2 with eigenvalues $\{-1\}$ or $\{-1,-2\}$. The sum of the orders of the resulting individual detectors is 32 , but the resulting global detector $R(s)$ has McMillan degree 6. Interestingly, the "least order" detector computed in [13] has order 14.

\section{Computational Aspects}

For the numerical computations, state space representation based algorithms have been developed to serve as a basis for robust software implementations. For this 
purpose, a state space realization of (1) is employed

$$
\begin{aligned}
& \dot{x}(t)=A x(t)+B_{u} u(t)+B_{d} d(t)+B_{f} f(t) \\
& y(t)=C x(t)+D_{u} u(t)+D_{d} d(t)+D_{f} f(t)
\end{aligned}
$$

with the $n$-dimensional state vector $x(t)$. The corresponding TFMs of the model in (1) are

$$
\begin{aligned}
& G_{u}(s)=C(s I-A)^{-1} B_{u}+D_{u} \\
& G_{d}(s)=C(s I-A)^{-1} B_{d}+D_{d} \\
& G_{f}(s)=C(s I-A)^{-1} B_{f}+D_{f}
\end{aligned}
$$

The FD Synthesis Procedure to design scalar output residual generators with least dynamical orders can be performed using the numerically sound computational approach proposed recently in [11]. This approach represents an enhancement of the minimal dynamic covers techniques introduced in [7], by employing Type I dynamic covers (instead of Type II covers) to achieve the maximal order reduction of the resulting detector. A basic computational ingredient to perform Step 1 is a reliable numerical algorithm to compute least order rational nullspaces of rational matrices using state-space methods [7]. The main computation in this algorithm is the orthogonal reduction of the system pencil matrix of the realization of $G(s)$ in (5) to a Kronecker-like form, from which, practically without any additional computation, a least order rational nullspace basis can be obtained. The existence conditions of the solution (6) can be easily checked using the outcome of the nullspace computation algorithm [11]. The least order fault detector at Step 2 can be obtained by selecting an appropriate linear combination of the basis vectors by eliminating non-essential dynamics using Type I dynamic covers based order reduction $[11,14]$. To perform Step 3, stable coprime factorization techniques can be used for which reliable numerical algorithms based on pole assignment techniques are available [15].

The efficient implementation of the enhanced FDI Synthesis Procedure requires an explicit updating of a preliminary design (16). State space realization based computations of $N_{f}(s)$ in (13) as well as of the resulting least order detectors $R^{i}(s)$ in (16) are described in [12]. Remarkably, the matrices of the underlying state space realizations of $N_{f}(s)$ can be obtained using exclusively orthogonal transformations on the system matrices of the original state space realization (17). By using these updating techniques, any need to determine minimal realizations (e.g., in (13)) has been practically eliminated.

For all underlying numerical computations, robust numerical software is available in the DESCRIPTOR SYSTEMS Toolbox [16]. This software underlies the implementation of a first version of a the FAULT DETECTION Toolbox [3], where several tools are available to solve the main classes of fault detection problems. The recently developed enhancements have been implemented in a new function fdsyn which is fully documented in [17]. 


\section{Monitoring Actuator Failures}

The monitoring of primary actuator failures of an aircraft is of paramount importance for the safe operation and for the continuous situation awareness of the pilots. In this section we address the fault detection and isolation of all FTC relevant actuator failures by combining component level and system level fault monitoring techniques. The main goal of our analysis is to prove the feasibility of a complete fault diagnosis system capable of localizing individual or simultaneous actuator/surface faults.

For our study we consider the Boeing 747-100/200 aircraft for which a high fidelity nonlinear simulation model with a full set of control surfaces is available. This model with 11 primary control surface actuators (4 elevators, 1 stabilizer, 4 ailerons, 2 rudders) has been set up within the GARTER AG16 as a benchmark for FTC studies. The original model [18] with only pilot inputs has been used in several fault detection studies [2], with focus on various aspects mentioned in Section 1.

For the Boeing 747-100/200 aircraft several fault scenarios are of particular interest. For example, the ability to detect single primary actuator faults is of critical importance, since it can be seen as part of the aircraft specification according to the requirements of FAA/FAR and EASA/CS. Thus a minimum request from the FTC perspective is the requirement that for modern aircraft design, no single failure leads to a catastrophic consequence.

Simultaneous faults can also occur, especially when multiple surface damages occur. The detection and isolation of simultaneous faults requires a more involved residual generation system and also the availability of a sufficiently large number of measurements. Although surface angle sensors can be installed on each control surface, an interesting aspect is to determine the minimum number of sensors necessary to completely solve the fault isolation problem. We give an answer to this problem by combining component level and system level fault monitoring.

The main goal of our study of detectability and isolability of actuator/surface faults was to demonstrate the feasibility of FDI for a complete set of faults. The full isolation requires placing a minimum number of additional surface angle sensors. An interesting result of our study also reveals the best achievable isolation capabilities in the absence of additional sensors.

Only the nominal case is studied corresponding to a normal cruise flight. The results obtained, consisting of several residual generators and the corresponding faultto-residual filter specifications, can serve as meaningful specifications for a more realistic design where input/output noise and uncertainties in the model parameters and flight conditions are also addressed. Finding the minimal number of additional sensors allowing the isolation of all surface faults is one of the main achievements of this study.

In what follows, we show first the capabilities of component level monitoring, which is traditionally used on present day aircraft. The intrinsic limitations of this approach, for example, to detect surface failures leading to loss of effectiveness, require addressing the FDIP using system level monitoring. However, the system level approach has its own limitations due to the restricted number of available measure- 
ments, therefore full FDI is not possible unless additional surface sensors are used. As can be easily guessed, the final solution of the FDIP is a combination of both approaches by employing a minimal number of sensors.

\subsection{Component Level Monitoring}

Typically actuators are modelled as first order linear systems which together with the corresponding control surfaces have transfer functions of the form

$$
g_{u}(s)=\frac{K}{s+K}
$$

Here the value of $K$ is determined taking into account the physical rate limits of the respective surface, and represents an average value applicable to all flight conditions. Typical choices for the Boeing actuators are: $37 /(s+37)$ for the elevators, $0.5 /(s+0.5)$ for the stabilizer, $50 /(s+50)$ for the ruders and ailerons. The task of the fault detection at the actuator level is to identify typical actuator faults like 'stuck actuator' (also called lock-in place failure), 'actuator runaway' (also called hard-over failure), 'free-play' (also called float-type failure), or loss of actuator effectiveness. In what follows we discuss some aspects of fault detection and isolation for a generic actuator.

Consider the actuator model (18) for which we would like to design a fault detector able to identify the fault types mentioned previously. For this purpose, a simple detector which estimates the deviation of surface position on the basis of measured control surface position and commanded control surface position is given by the simple observer-like structure

$$
R(s)=\left[1-g_{u}(s)\right]
$$

Note that the dynamics of filter can be arbitrarily assigned by replacing $R(s)$ with $m(s) R(s)$, where $m(s)$ is an arbitrary stable transfer function.

With such a detector, an actuator fault can be easily detected by checking the condition $r(t) \neq 0$. The stationary value of the residual signal $r(\infty)$ can also be used to estimate the actual DC-gain of the actuator, say $g_{0}$, and thus the actuator effectiveness. Since $g_{0}=1-r(\infty)$, in the fault-free case we have $g_{0}=1$. DC-gain values in the range $[0,1]$ indicate a loss of actuator effectiveness with a zero gain indicating 'free-play'. Values outside this domain indicates either a 'stuck actuator' in a certain position or even an 'actuator runaway' (i.e., stuck in an extreme position).

The main weakness of this simple fault detection scheme is that it does not work properly in the case of surface position sensor failures. This lack of reliability against combined actuator and sensor failures could be a source of false alarms. Another potential problem is when the actuator is fault free but the corresponding control surface is damaged. The associated loss of effectiveness of the actuation/control surface system can not be detected in this way. 
A typical approach to overcome the first weakness is to add hardware redundancy by increasing the number of sensors to a level which ensures a satisfactory reliability of measurements. A standard approach is to use three sensors in a voting logic for validity checking. This is the minimum hardware redundancy to guarantee the reliability of monitoring. Interestingly, using model based fault detection techniques, it is possible to obtain practically the same level of confidence by using only two sensors (the model based approach provides a third 'virtual' sensor).

The actuator system with two identical sensors is described by the transferfunction matrix

$$
G_{u}(s)=\left[\begin{array}{l}
1 \\
1
\end{array}\right] g_{u}(s)
$$

The fault TFM corresponding to the actuator fault $f_{1}$ and two sensor faults $f_{2}$ and $f_{3}$ is

$$
G_{f}(s)=\left[G_{u}(s) I_{2}\right]
$$

A possible least order detector for this setup can be chosen as

$$
R(s)=\left[\begin{array}{ccc}
1 & -1 & 0 \\
0 & 1 & -g_{u}(s) \\
1 & 0 & -g_{u}(s)
\end{array}\right]
$$

and can be realized as a first order system. The resulting fault detection system achieves the following fault signature

$$
S=\left[\begin{array}{lll}
0 & 1 & 1 \\
1 & 0 & 1 \\
1 & 1 & 0
\end{array}\right]
$$

Assuming that the actuator fault and sensor faults occur one at a time, this influence structure provides a complete isolation of a single fault by using the following isolation logic:

- actuator fault occurred if $r_{1}=0, r_{2} \neq 0$, and $r_{3} \neq 0$;

- first sensor failed if $r_{1} \neq 0, r_{2}=0$, and $r_{3} \neq 0$;

- second sensor failed if $r_{1} \neq 0, r_{2} \neq 0$, and $r_{3}=0$.

In this way, the occurrence of each fault can be reliably detected. For fault identification, the information provided by either residual signal $r_{1}$ or $r_{2}$ can be employed.

To address the second aspect of loss of control surface effectiveness a system level analysis could be appropriate (see next section).

For component level diagnosis more detailed actuator models can be used, by explicitly modelling the dynamics of all actuator components. Such an approach based on physical parametric models is also suitable for health monitoring purposes.

Another application of potential interest is to detect the so-called 'oscillatory failure' (e.g., of a rudder) as a result of limit cycle oscillations. This type of failure can trigger an aeroelastic resonance behavior of the aircraft with unacceptably high loads. To identify this type of faults, the detection scheme above can be supple- 
mented with an additional signal analysis based oscillation detection system (e.g., sub-band filtering followed by Fourier analysis).

\subsection{System Level Monitoring}

The monitoring of actuator faults at the system level is primarily intended to increase overall aircraft safety by detecting fault categories which can not be handled by the usual component level monitoring. Such faults are, for example, the loss of efficiency of control surfaces due to possible structural damage or as a result of icing.

The study of the nominal case has as its main purpose getting a clear understanding of the intrinsic limitations in solving the FDIP in an idealized situation. Furthermore, the achieved fault-to-residual specifications can serve as reference models for a model-matching formulation of the FDIP [19], where system variabilities (parametric, flight conditions) are fully considered.

Actuator fault diagnosis for the whole aircraft can be done in several ways. An approach advocated by several authors is to use so-called multi-models describing the aircraft in normal flight conditions as well as in several faulty situations. A bank of model detection filters can be designed to ensure a desired model-to-residual signature allowing the application of simple decision logic to identify the current model (normal or faulty). The main advantage of this approach is its simplicity, both because of a simple design of the detectors as well as because of the simple residual evaluation scheme. The main disadvantage is the need for a large number of models (and thus detectors) to cover many faults and combinations of faults. Moreover, different levels of actuator efficiency loss are usually represented as separate models, thus making the number of necessary detectors increase exponentially.

The approach we follow in our study is to model actuator faults as additive disturbances. The linearized fault model of the aircraft corresponding to a given set of parameter values and a specific flight condition (e.g., straight-and-level flight) has the standard input-output form (1) and the detector is designed in the filter form (2). The linearized models which have been employed were determined using the nominal values of the parameters in Table 5.2. In what follows we summarize the results of designing fault detectors for the nominal case.

The longitudinal and full order linearized state space models of the aircraft are given in Appendices A and B. These models correspond to the following parameter values: mass $=317,000 \mathrm{~kg}$, center of gravity coordinates: $X_{c g}=25 \%, Y_{c g}=0, Z_{c g}=$ 0 . The chosen flight condition is a straight-and-level flight at altitude $600 \mathrm{~m}$, with a speed of $92.6 \mathrm{~m} / \mathrm{s}$, with a flap setting at $20^{\circ}$ and with landing gear up. For more details on the employed model see [18]. 
Table 1 Definition of variables and trim condition

\begin{tabular}{lll}
\hline Variable & Nominal Value & Range \\
\hline Altitude & $600 \mathrm{~m}(2000 \mathrm{ft})$ & {$[0,1000]$} \\
Air speed & $92.6 \mathrm{~m} / \mathrm{s}$ & {$[85,135]$} \\
Landing gear & up & \\
Mass[kg] & 317,000 & {$[263,000,320,000]$} \\
$X_{c g}$ & $25 \%$ & {$[22 \%, 28 \%]$} \\
$Y_{c g}[m]$ & 0 & {$[-1,1]$} \\
$Z_{c g}[m]$ & 0 & {$[-1,1]$} \\
Flight path angle $(\gamma)$ & $0^{o}$ & \\
Flap setting & $20^{\circ}$ & \\
\hline
\end{tabular}

\subsection{Pitch Axis Fault Monitoring}

To detect elevator and/or stabilizer faults, we use the longitudinal aircraft model in state-space form (17), where the matrices $A, B_{u}, C$, and $D_{u}$ are defined in Appendix A. The elevator and stabilizer fault inputs are defined as

$$
f=\left[\begin{array}{r}
f_{1} \\
f_{2} \\
f_{3} \\
f_{4} \\
f_{5}
\end{array}\right]\left(\begin{array}{r}
\text { right inner elevator fault }[\mathrm{rad}] \\
\text { left inner elevator fault }[\mathrm{rad}] \\
\text { right outer elevator fault }[\mathrm{rad}] \\
\text { left outer elevator fault }[\mathrm{rad}] \\
\text { stabilizer fault }[\mathrm{rad}]
\end{array}\right)
$$

and thus $B_{f}=B_{u}(:, 1: 5)$ and $D_{f}=D_{u}(:, 1: 5)$. For this study of the nominal case we consider no disturbance inputs for the model.

The achievable fault signature is

$$
S=\left[\begin{array}{rrrrr}
1 & 1 & 1 & 1 & 1 \\
0 & 0 & 1 & 1 & 1 \\
1 & 1 & 0 & 0 & 1 \\
1 & 1 & 1 & 1 & 0 \\
-1 & -1 & 0 & 0 & 0 \\
0 & 0 & -1 & -1 & 0 \\
0 & 0 & 0 & 0 & -1
\end{array}\right]
$$

From the last three lines of $S$ it can be observed that the isolation of faults grouped in three groups $\left(f_{1}, f_{2}\right),\left(f_{3}, f_{4}\right)$ and $f_{5}$ is achievable, although all groups are only weakly detectable.

System level monitoring can be used as a complementary tool to device level monitoring in the case when sensor fault monitoring is not additionally provided. The simplest fault detection task is to determine if any actuator fault in the pitch axis has occurred. This comes down to the design of a fault detector achieving the trivial signature corresponding to the first row of $S$ 


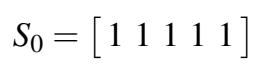

by using the lowest order dynamics. To design such a detector, the function fdsyn has been used. Using the least order design option, a first order residual generator can be determined. The resulting fault-to-residual dynamics are

$$
R_{f}(s)=\left[\begin{array}{lllll}
\frac{10}{s+10} & \frac{10}{s+10} & \frac{10.43}{s+10} & \frac{10.43}{s+10} & \frac{-5.188 s+58.45}{s+10}
\end{array}\right]
$$

If we would like to isolate elevator and stabilizer faults, only the following choice of the signature matrix is achievable

$$
S_{1}=\left[\begin{array}{rrrrr}
1 & 1 & 1 & 1 & 0 \\
0 & 0 & 0 & 0 & -1
\end{array}\right]
$$

with the second row having only a weak detectability structure. If we assume that elevator and stabilizer faults can not simultaneously occur, we can achieve elevator and stabilizer fault isolation by using the specification matrix

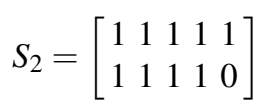

To isolate $\left(f_{1}, f_{2}, f_{3}, f_{4}\right)$ and $f_{5}$ the following decision logic can be used:

- elevator fault occurred if $r_{2} \neq 0$;

- stabilizer fault occurred if $r_{1} \neq 0$ and $r_{2}=0$.

A residual generator achieving the above specification can be obtained as a bank of two detectors using the function fdsyn. Using the least order design option, two first order detectors can be determined, leading to a residual generator of total order 2.

Provided we can assume that the groups of faults $\left(f_{1}, f_{2}\right),\left(f_{3}, f_{4}\right)$ and $f_{5}$ do not simultaneously occur, the achievable specification

$$
S_{3}=\left[\begin{array}{lllll}
0 & 0 & 1 & 1 & 1 \\
1 & 1 & 0 & 0 & 1 \\
1 & 1 & 1 & 1 & 0
\end{array}\right]
$$

can be used for weak isolation using the following decision logic:

- inner elevator fault occurred if $r_{1}=0, r_{2} \neq 0$, and $r_{3} \neq 0$;

- outer elevator fault occurred if $r_{1} \neq 0, r_{2}=0$, and $r_{3} \neq 0$;

- stabilizer fault occurred if $r_{1} \neq 0, r_{2} \neq 0$, and $r_{3}=0$.

Using the least order design option, three first order detectors can be obtained using the function fdsyn leading to a detector of total order 3. Note that without the least order design option, a detector of total order 10 results, while using the standard observer based approach (see for example [20]), a detector of total order 15 is to be expected. The resulting fault-to-residual dynamics are 


$$
R_{f}(s)=\left[\begin{array}{ccccc}
0 & 0 & \frac{10}{s+10} & \frac{10}{s+10} & \frac{862.7 s-1889}{s+10} \\
\frac{10}{s+10} & \frac{10}{s+10} & 0 & 0 & \frac{-835.1 s+2028}{s+10} \\
\frac{10}{s+10} & \frac{10}{s+10} & \frac{10.74}{s+10} & \frac{10.74}{s+10} & 0
\end{array}\right]
$$

The step responses associated with the faults are presented in Fig. 1.

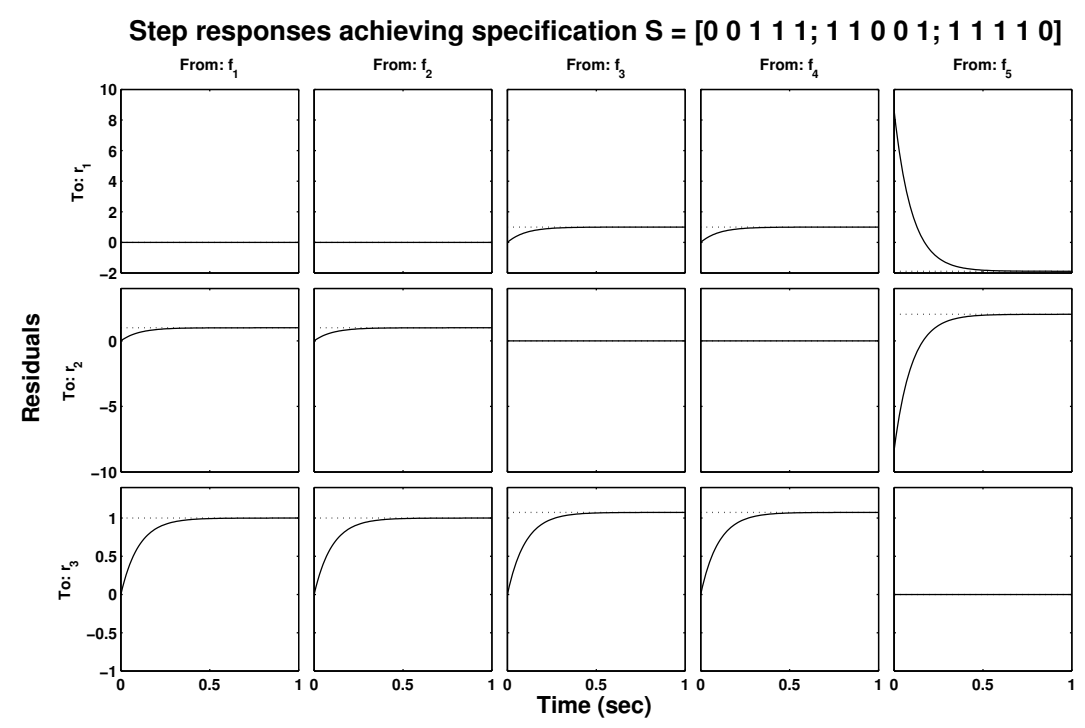

Fig. 1 Step responses from the faults: $f_{1}=1, \ldots, f_{4}=1, f_{5}=0.01$.

A more realistic setting is to add actuator dynamics to each input actuator-surface channel [2]. As already mentioned, the elevator dynamics can be approximated by transfer functions of the form $37 /(s+37)$, while for the stabilizer dynamics we take $0.5 /(s+0.5)$ as suggested in [2]. The resulting model has now order 10 and we can achieve the same fault signature with a bank of three detectors of total order 6 . The step responses from the faults are presented in Fig. 2.

Further enhancement of fault isolation is possible by employing direct measurements of surface positions. For example, with a single additional measurement of the stabilizer surface angle it is possible to achieve the signature specification

$$
S_{4}=\left[\begin{array}{lllll}
1 & 1 & 0 & 0 & 0 \\
0 & 0 & 1 & 1 & 0 \\
0 & 0 & 0 & 0 & 1
\end{array}\right]
$$




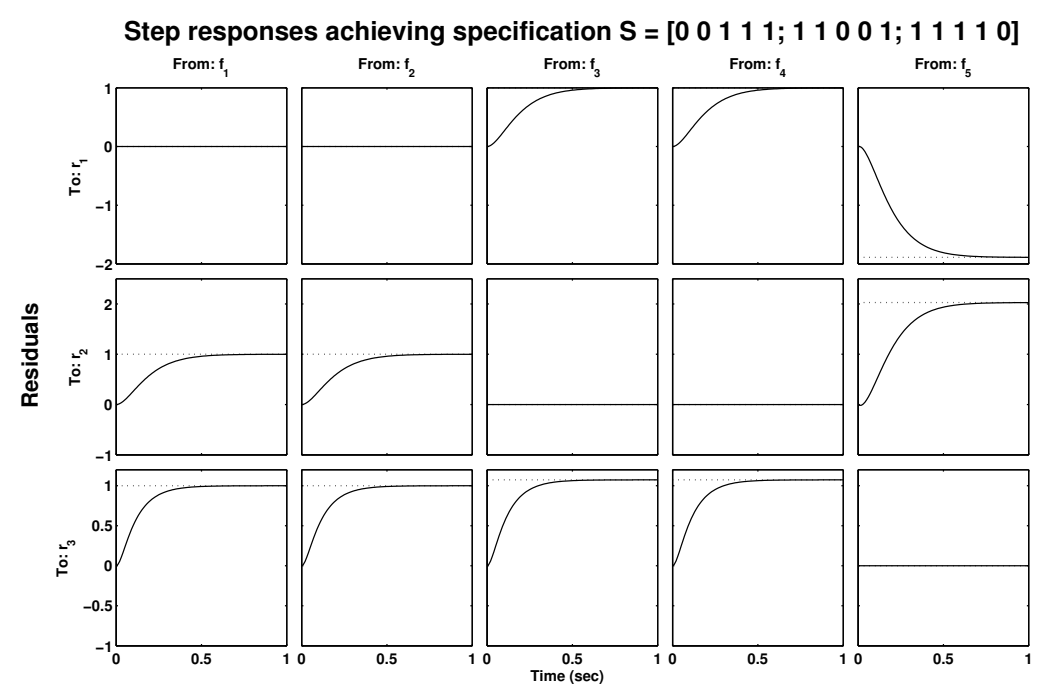

Fig. 2 Step responses from the faults (included actuator dynamics): $f_{1}=1, \ldots, f_{4}=1, f_{5}=0.01$.

and thus to isolate the inner elevator, the outer elevator and the stabilizer faults. The above specification can be achieved using a bank of three detectors of total order 5 . The step responses from the faults are presented in Fig. 3.

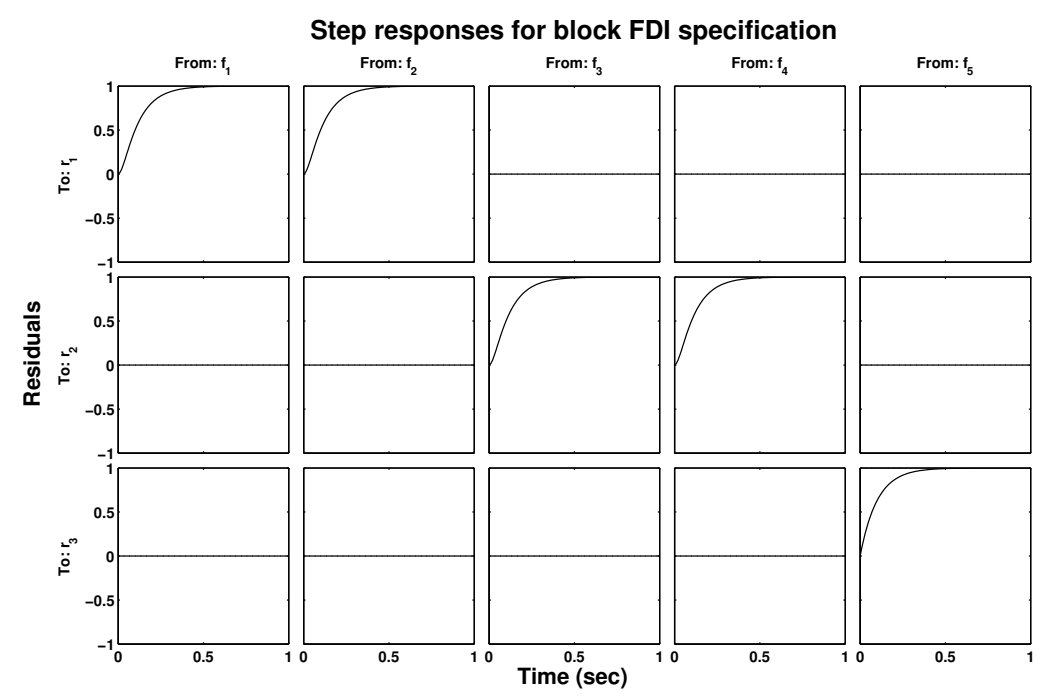

Fig. 3 Step responses from the faults with stabilizer angle measurement. 
Finally, for complete fault isolation it is to be expected that measurements from all surfaces are necessary. Solving the fault detection and isolation problem corresponds to achieve the specification $S_{5}=I_{5}$ using the function fdsyn or employing directly the specially devised function $f d i$, available in the FAULT DETECTION toolbox [3]. This latter function is based on the method proposed in [9]. Using this function, we obtain a detector of order 5 which solves the complete fault detection and isolation problem. Interestingly, this detector is the same as that one obtained by using single surface monitoring schemes. This remarkable result also illustrates the real strengths of the recently developed minimal degree design techniques [9]. In contrast, the methods traditionally used (e.g., using a bank of 5 observer based detectors [20]) could lead to detectors of total order up to 70 in the case when actuator dynamics are included.

Interestingly, complete isolation can also be achieved by choosing a minimal number of three surface measurements: two from the left elevators and one from the stabilizer. The resulting bank of five detectors has a total order of 7 and the resulting fault-to-residual TFM is

$$
R_{f}(s)=\operatorname{diag}\left(\frac{10}{s+10}, \frac{370}{s^{2}+47 s+370}, \frac{10}{s+10}, \frac{370}{s^{2}+47 s+370}, \frac{10}{s+10}\right)
$$

The step responses from the faults are presented in Fig. 4.

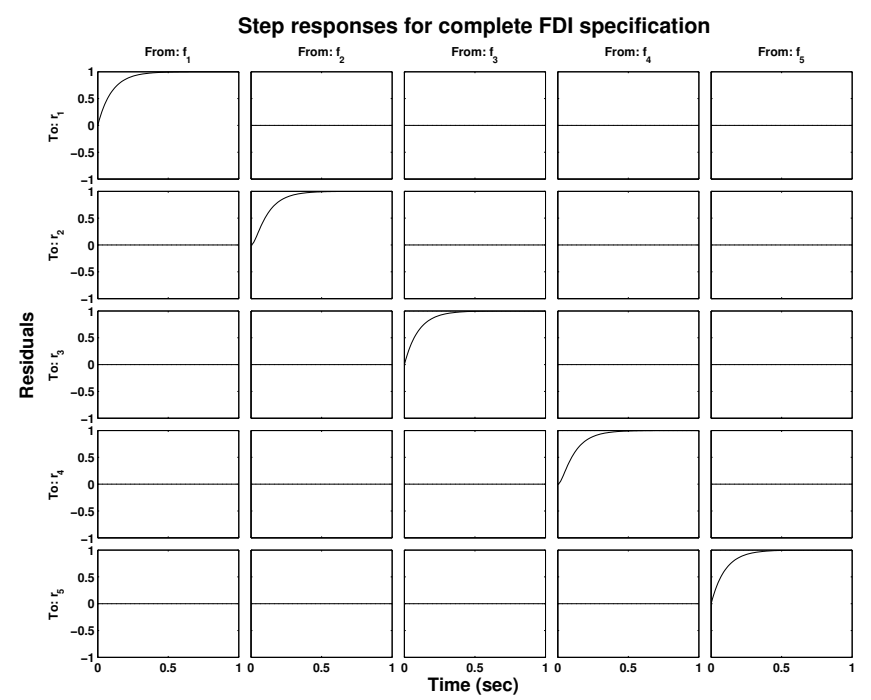

Fig. 4 Step responses from the faults with left elevators and stabilizer angles measurements. 


\subsection{Gear and Roll Axes Fault Monitoring}

To detect rudder and/or aileron faults, we consider the full order $(n=10)$ aircraft model in state-space form (17). The definition of state, input and output variables and the corresponding state space matrices are given in Appendix B. The aileron and rudder fault inputs are defined as

$$
f=\left[\begin{array}{l}
f_{1} \\
f_{2} \\
f_{3} \\
f_{4} \\
f_{5} \\
f_{6}
\end{array}\right]\left(\begin{array}{r}
\text { right inner aileron fault }[\mathrm{rad}] \\
\text { left inner aileron fault }[\mathrm{rad}] \\
\text { right outer aileron fault }[\mathrm{rad}] \\
\text { left outer aileron fault }[\mathrm{rad}] \\
\text { upper rudder fault }[\mathrm{rad}] \\
\text { lower rudder fault }[\mathrm{rad}]
\end{array}\right)
$$

and thus $B_{f}$ and $D_{f}$ are formed from the columns $\{1,2,3,4,10,11\}$ of $B_{u}$ and $D_{u}$, respectively.

For the two inner aileron faults $\left\{f_{1}, f_{2}\right\}$, two outer aileron faults $\left\{f_{3}, f_{4}\right\}$, and two rudder faults $\left\{f_{5}, f_{6}\right\}$, the FDIP with the fault signature

$$
S_{1}=\left[\begin{array}{llllll}
1 & 1 & 0 & 0 & 0 & 0 \\
0 & 0 & 1 & 1 & 0 & 0 \\
0 & 0 & 0 & 0 & 1 & 1
\end{array}\right]
$$

is achievable using a bank of three detectors with global order 3 . The resulting faultto-residual TFM is

$$
R_{f}(s)=\left[\begin{array}{cccccc}
\frac{10}{s+10} & \frac{10}{s+10} & 0 & 0 & 0 & 0 \\
0 & 0 & \frac{10}{s+10} & \frac{10}{s+10} & 0 & 0 \\
0 & 0 & 0 & 0 & \frac{11.85}{s+10} & \frac{10}{s+10}
\end{array}\right]
$$

The step responses from the faults are presented in Fig. 5.

We include now the actuator models and add three surface angle sensors for the two right ailerons and for the upper ruder. With this sensor location the complete FDIP with $S_{2}=I_{6}$ can be solved to isolate all aileron and rudder failures. The resulting detector has order 9 and the achieved fault-to-residual TFM is

$$
\begin{gathered}
R_{f}(s)=\operatorname{diag}\left(\frac{10}{s+10}, \frac{100}{s^{2}+20 s+100}, \frac{10}{s+10},\right. \\
\left.\frac{100}{s^{2}+20 s+100}, \frac{10}{s+10}, \frac{-0.0002566 s+100}{s^{2}+20 s+100}\right)
\end{gathered}
$$

The step responses from the faults are presented in Fig. 6. 


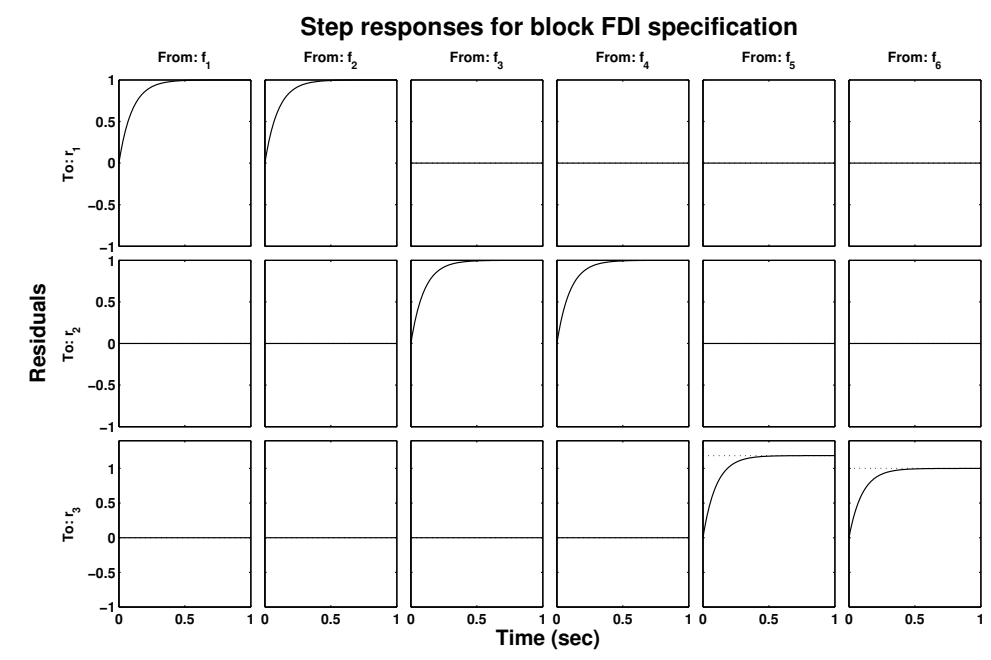

Fig. 5 Step responses from the aileron and rudder faults.

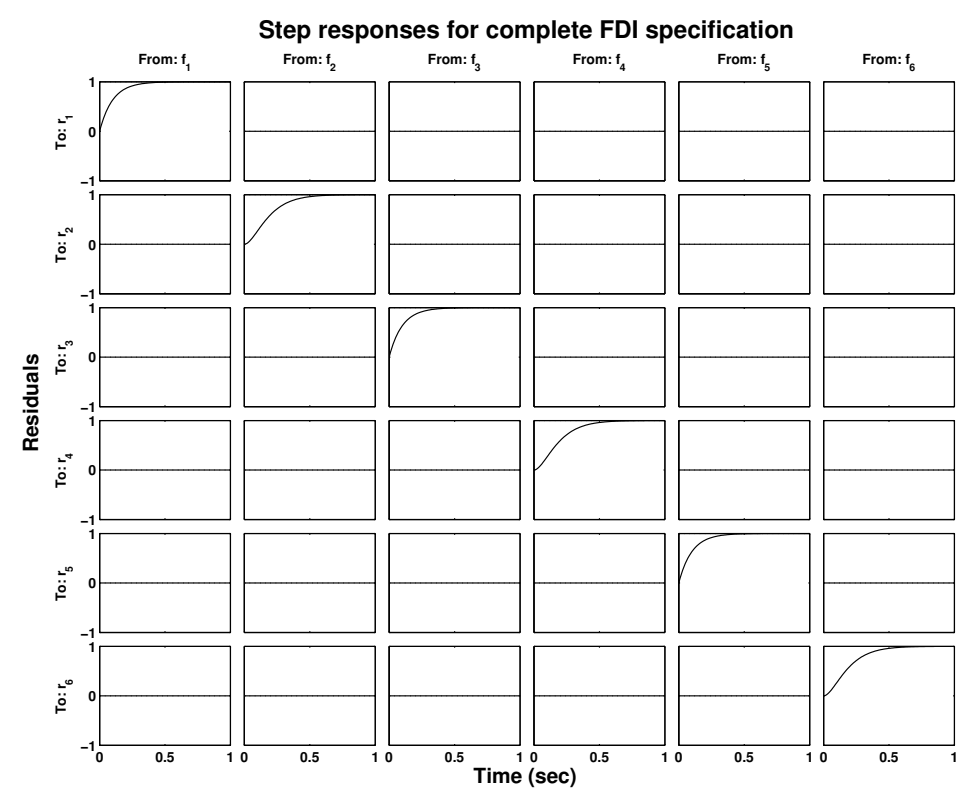

Fig. 6 Step responses from the aileron and rudder faults. 


\section{Summary of Achieved Results and Needs for Further Analysis}

The nominal design of residual generators which has been undertaken provides valuable insight into the nature of the FDIP for aircraft actuator failures, demonstrates the feasibility of complete fault isolation, and provides filter specifications which can be useful in a more realistic design of robust residual generators. The analysis which has been performed of the FDIP for a complete set of primary flight surfaces shows that a combination of component level monitoring with a system level monitoring, allows the solution of this problem for a set of 11 actuator/surface failures. Our study demonstrated the interesting fact that by appropriately locating a minimal number of 6 surface angle sensors, complete isolation of faults is possible. The resulting orders of the residual generators are surprisingly low: order 7 for pitch axis monitoring and 9 for gear/roll axis monitoring. These figures lower to 3 and 3, respectively, if no actuator models are included in the design.

By using the proposed least order detector design techniques implemented in reliable numerical software, a seamless switching among a large number of different sensor configurations was possible using a single global model of larger order. Interestingly, the reliability of the numerical algorithms which were employed allowed us, to recover the same simple results in the case when sensors are used for all surfaces, as those obtained working with each actuator/surface component individually.

For the complete solution of the FDIP, the following aspects still need careful consideration:

1. Surface angle sensor faults. To achieve complete reliability of the fault monitoring system, it is important to also consider possible faults in the surface angle sensors. For example, by adding sensors to all surfaces, the complete isolation of all actuator faults is possible, while additionally the isolation of a sensor fault (e.g., stabilizer angle sensor) can be achieved. With three sensors (e.g., two for left elevators and one for stabilizer), to achieve the isolation of one sensor fault, we have to assume that sensor and actuator faults do not occur simultaneously. A complete analysis of sensor location and assignment aspects is important for practical applications (see also Part II of [21] for a recent survey).

2. Robustness against noisy inputs and noisy measurements. The effect of noisy inputs and noisy measurements must be considered in a realistic design. Typical noisy inputs for aircraft are gust turbulences, which can be taken into account by feeding white noise into the system via stable and minimum-phase Dryden spectra filters. Colouring filters driven by white noise can be used to model noise in sensor measurements. For further details see [2] and the literature cited therein.

3. Robustness against parametric uncertainties. The robustness of the designed detectors against parametric uncertainties is important for practical applicability. Typical uncertain parameters to be considered for robustness studies are mass, the coordinates of the center of gravity, as well as flight conditions (speed, altitude). There are many possibilities to enforce the robustness of the designed detectors [22] and this challenging aspect will be considered in further studies. The results provided in this work can be seen as realistic specifications of what can be aimed to be achieved in the most favorable situation. 


\section{References}

1. I. Szászi, S. Ganguli, A. Marcos, G. J. Balas, and J. Bokor. Application of FDI to a nonlinear Boeing-747 aircraft. Proc. Mediterranean Conference on Control and Automation, Lisbon, Portugal, 2002.

2. A. Marcos, S. Ganguli, and G. J. Balas. An application of $\mathscr{H}_{\infty}$ fault detection and isolation to a transport aircraft. Control Engineering Practice, 13:105-119, 2005.

3. A. Varga. A fault detection toolbox for Matlab. Proc. of CACSD'06, Munich, Germany, 2006.

4. X. Ding and P. M. Frank. Frequency domain approach and threshold selector for robust modelbased fault detection and isolation. Proc. of IFAC Symposium SAFEPROCESS 1991, BadenBaden, Germany, 1991.

5. M. Nyberg. Criterions for detectability and strong detectability of faults in linear systems. Int. J. Control, 75:490-501, 2002.

6. E. Frisk and M. Nyberg. A minimal polynomial basis solution to residual generation for fault diagnosis in linear systems. Automatica, 37:1417-1424, 2001.

7. A. Varga. On computing least order fault detectors using rational nullspace bases. Proc. of IFAC Symp. SAFEPROCESS'2003, Washington D.C., 2003.

8. J. Gertler. Fault Detection and Diagnosis in Engineering Systems. Marcel Dekker, New York, 1998.

9. A. Varga. New computational approach for the design of fault detection and isolation filters. In M. Voicu (Ed.), Advances in Automatic Control, vol. 754 of The Kluwer International Series in Engineering and Computer Science, pp. 367-381, Kluwer Academic Publishers, 2004.

10. J. Gertler. Designing dynamic consistency relation for fault detection and isolation. Int. J. Control, 73:720-732, 2000.

11. A. Varga. On designing least order residual generators for fault detection and isolation. Proc. 16th Internat. Conf. on Control Systems and Computer Science, Bucharest, Romania, pp. 323$330,2007$.

12. A. Varga. On computing nullspace bases - a fault detection perspective. Proc. IFAC 2008 Word Congress, Seoul, Korea, 2008.

13. Z. Yuan, G. C. Vansteenkiste, and C. Y. Wen. Improving the observer-based FDI design for efficient fault isolation. Int. J. Control, 68(1):197-218, 1997.

14. A. Varga. Reliable algorithms for computing minimal dynamic covers. Proc. of CDC'2003, Maui, Hawaii, 2003.

15. A. Varga. Computation of coprime factorizations of rational matrices. Lin. Alg. \& Appl., 271:83-115, 1998.

16. A. Varga. A DesCriptor Systems toolbox for Matlab. Proc. CACSD'2000 Symposium, Anchorage, Alaska, 2000.

17. A. Varga. Linear FDI-Techniques and Software Tools. FAULT Detection Toolbox V0.8 - Technical Documentation, German Aerospace Center (DLR), Institute of Robotics and Mechatronics, 2008.

18. A. Marcos and G. J. Balas. A Boeing 747-100/200 Aircraft Fault Tolerant and Fault Diagnostic Benchmark. Technical Report AEM-UoM-2003-1, Department of Aerospace and Engineering Mechanics, University of Minnesota, USA, 2003.

19. A. Varga. Numerically reliable methods for optimal design of fault detection filters. Proc. of CDC'05, Seville, Spain, 2005.

20. R. J. Patton and M. Hou. Design of fault detection and isolation observers: a matrix pencil approach. Automatica, 34(9):1135-1140, 1998.

21. C. Commault and J.-M. Dion. Sensor location for diagnosis in linear systems: a structural analysis. IEEE Trans. Automat. Control, 52:155-169, 2007.

22. J. Chen and R. J. Patton. Robust Model-Based Fault Diagnosis for Dynamic Systems. Kluwer Academic Publishers, London, 1999. 


\section{Appendix A Linearized longitudinal model}

\section{Definition of variables}

For the trim conditions defined for the nominal values in Table 5.2, the corresponding linearized nominal longitudinal state space model of the Boeing 747 has the form

$$
\begin{aligned}
& \dot{x}(t)=A x(t)+B_{u} u(t) \\
& y(t)=C x(t)+D_{u} u(t)
\end{aligned}
$$

where the state, input and output variables are defined as follows:

$$
\begin{aligned}
& x=\left[\begin{array}{c}
\delta q \\
\delta V_{T A S} \\
\delta \alpha \\
\delta \theta \\
\delta h_{e}
\end{array}\right]=:\left(\begin{array}{r}
\text { pitch rate }[\mathrm{rad} / \mathrm{s}] \\
\text { true airspeed }[\mathrm{m} / \mathrm{s}] \\
\text { angle of attack }[\mathrm{rad}] \\
\text { pitch angle }[\mathrm{rad}] \\
\text { altitude }[\mathrm{m}]
\end{array}\right)
\end{aligned}
$$

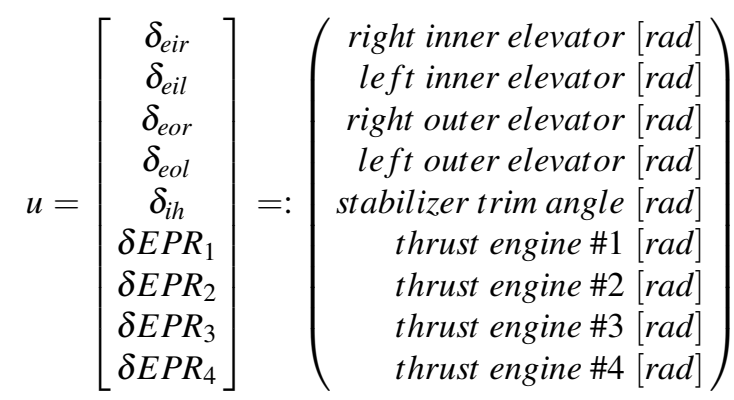

$$
\begin{aligned}
& y=\left[\begin{array}{c}
\delta \alpha \\
\delta \dot{V}_{T A S} \\
\delta \theta \\
\delta q \\
\delta V_{z} \\
\delta h_{e}
\end{array}\right]=:\left(\begin{array}{c}
\text { angle of attack }[\mathrm{rad}] \\
\text { acceleration }\left[\mathrm{m} / \mathrm{s}^{2}\right] \\
\text { pitch angle }[\mathrm{rad}] \\
\text { pitch rate }[\mathrm{rad} / \mathrm{s}] \\
\text { vertical velocity }[\mathrm{m} / \mathrm{s}] \\
\text { altitude }[\mathrm{m}]
\end{array}\right)
\end{aligned}
$$




\section{State-model matrices}

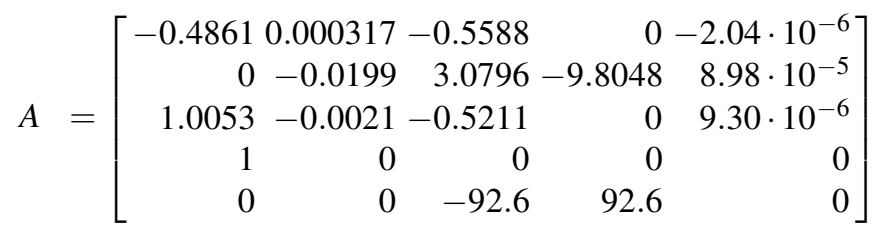

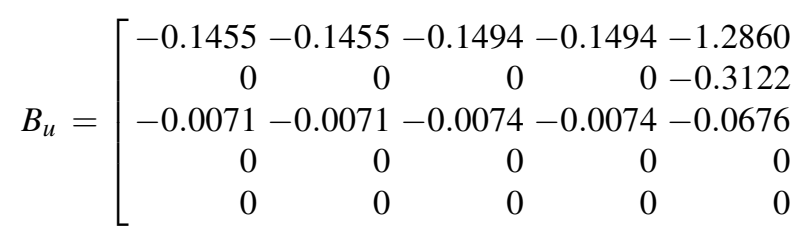

$$
\begin{aligned}
& \left.\begin{array}{llll}
0.0013 & 0.0035 & 0.0035 & 0.0013
\end{array}\right] \\
& \begin{array}{llll}
0.1999 & 0.1999 & 0.1999 & 0.1999
\end{array} \\
& -0.0004-0.0004-0.0004-0.0004 \\
& \left.\begin{array}{llll}
0 & 0 & 0 & 0 \\
0 & 0 & 0 & 0
\end{array}\right] \\
& C=\left[\begin{array}{rrrrr}
0 & 0 & 1 & 0 & 0 \\
0 & -0.0199 & 3.0796 & -9.8048 & 8.98 \cdot 10^{-5} \\
0 & 0 & 0 & 1 & 0 \\
1 & 0 & 0 & 0 & 0 \\
0 & 0 & -92.6 & 92.6 & 0 \\
0 & 0 & 0 & 0 & 1
\end{array}\right]
\end{aligned}
$$

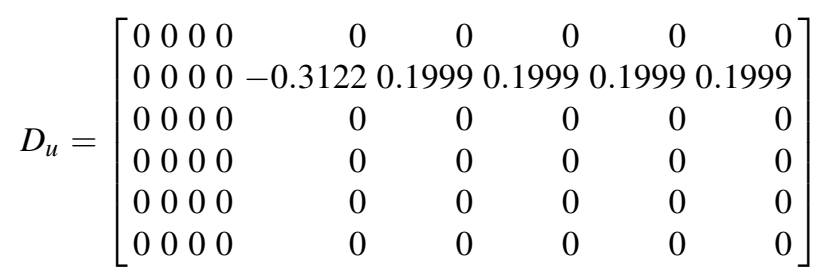




\section{Appendix B Linearized full order model}

\section{Definition of variables}

The trim conditions are defined for the nominal values specified in Table 5.2. The state, control and output variables are defined as follows:

$$
\begin{aligned}
& x=\left[\begin{array}{c}
\delta p \\
\delta q \\
\delta r \\
\delta V_{T A S} \\
\delta \alpha \\
\delta \beta \\
\delta \phi \\
\delta \theta \\
\delta \psi \\
\delta h_{e}
\end{array}\right]=:\left(\begin{array}{r}
\text { roll rate }[\mathrm{rad} / \mathrm{s}] \\
\text { pitch rate }[\mathrm{rad} / \mathrm{s}] \\
\text { yaw rate }[\mathrm{rad} / \mathrm{s}] \\
\text { true airspeed }[\mathrm{m} / \mathrm{s}] \\
\text { angle of attack }[\mathrm{rad}] \\
\text { sideslip angle }[\mathrm{rad}] \\
\text { roll angle }[\mathrm{rad}] \\
\text { pitch angle }[\mathrm{rad}] \\
\text { yaw angle }[\mathrm{rad}] \\
\text { altitude }[\mathrm{m}]
\end{array}\right) \\
& u=\left[\begin{array}{c}
\delta_{\text {air }} \\
\delta_{\text {ail }} \\
\delta_{a o r} \\
\delta_{a o l} \\
\delta_{\text {eir }} \\
\delta_{\text {eil }} \\
\delta_{\text {eor }} \\
\delta_{\text {eol }} \\
\delta_{i h} \\
\delta_{r u} \\
\delta_{r l} \\
\delta E P R_{1} \\
\delta E P R_{2} \\
\delta E P R_{3} \\
\delta E P R_{4}
\end{array}\right]=:\left(\begin{array}{r}
\text { right inner aileron }[\mathrm{rad}] \\
\text { left inner aileron }[\mathrm{rad}] \\
\text { right outer aileron }[\mathrm{rad}] \\
\text { left outer aileron }[\mathrm{rad}] \\
\text { right inner elevator }[\mathrm{rad}] \\
\text { left inner elevator }[\mathrm{rad}] \\
\text { right outer elevator }[\mathrm{rad}] \\
\text { left outer elevator }[\mathrm{rad}] \\
\text { stabilizer trim angle }[\mathrm{rad}] \\
\text { upper rudder surface }[\mathrm{rad}] \\
\text { lower rudder surface }[\mathrm{rad}] \\
\text { thrust engine \#1 }[\mathrm{rad}] \\
\text { thrust engine \#2 }[\mathrm{rad}] \\
\text { thrust engine \#3 }[\mathrm{rad}] \\
\text { thrust engine \#4 }[\mathrm{rad}]
\end{array}\right) \\
& y=\left[\begin{array}{c}
\delta \alpha \\
\delta \dot{V}_{T A S} \\
\delta \theta \\
\delta q \\
\delta V_{z} \\
\delta h_{e} \\
\delta p \\
\delta r \\
\delta \beta \\
\delta V_{y} \\
\delta \phi
\end{array}\right]=:\left(\begin{array}{c}
\text { angle of attack }[\mathrm{rad}] \\
\text { acceleration }\left[\mathrm{m} / \mathrm{s}^{2}\right] \\
\text { pitch angle }[\mathrm{rad}] \\
\text { pitch rate }[\mathrm{rad} / \mathrm{s}] \\
z-\text { velocity }[\mathrm{m} / \mathrm{s}] \\
\text { altitude }[\mathrm{m}] \\
\text { roll rate }[\mathrm{rad} / \mathrm{s}] \\
\text { yaw rate }[\mathrm{rad} / \mathrm{s}] \\
\text { sideslip angle }[\mathrm{rad}] \\
y-\text { velocity }[\mathrm{m} / \mathrm{s}] \\
\text { roll angle }[\mathrm{rad}]
\end{array}\right)
\end{aligned}
$$


State-model matrices

$$
\begin{aligned}
& A=\left[\begin{array}{rrrrrrr}
-.8226 & 0 & 0.1666 & 0 & 0 & -1.4189 & 0.000471 \\
0 & -0.4861 & 0 & 0.000317 & -0.5588 & 0 & 0 \\
-.1303 & 0 & -0.0199 & 0 & 0 & 0.2387 & -0.00166 \\
0 & 0 & 0 & -0.0199 & 3.0796 & 0 & 0 \\
0 & 1.0053 & 0 & -0.0021 & -0.5211 & 0 & 0 \\
0.139 & 0 & -0.9867 & 0 & 0 & 0.0819 & 0.10505 \\
1 & 0 & 0.1265 & 0 & 0 & 0 & 0 \\
0 & 1 & 0 & 0 & 0 & 0 & 0 \\
0 & 0 & 1.008 & 0 & 0 & 0 & 0 \\
0 & 0 & 0 & 0 & -92.6 & 0 & 0
\end{array}\right. \\
& \left.\begin{array}{rrr}
0 & 0 & 0 \\
0 & 0 & -2.04 \cdot 10^{-6} \\
0 & 0 & 0
\end{array}\right] \\
& \begin{array}{rll}
-9.80480 & 0 & 8.98 \cdot 10^{-5}
\end{array} \\
& \begin{array}{lll}
0 & 0 & 9.30 \cdot 10^{-6}
\end{array} \\
& \begin{array}{lll}
0 & 0
\end{array} \\
& \begin{array}{ll}
0 & 0
\end{array} \\
& \begin{array}{ll}
0 & 0
\end{array} \\
& \begin{array}{ll}
0 & 0
\end{array} \\
& 92.60 \\
& C=\left[\begin{array}{rrrrrrrrrr}
0 & 0 & 0 & 0 & 1 & 0 & 0 & 0 & 0 & 0 \\
0 & 0 & 0 & -0.0199 & 3.0796 & 0 & 0 & -9.8048 & 0 & 8.98 \cdot 10^{-5} \\
0 & 0 & 0 & 0 & 0 & 0 & 0 & 10 & 0 & 0 \\
0 & 1 & 0 & 0 & 0 & 0 & 0 & 0 & 0 & 0 \\
0 & 0 & 0 & 0 & -92.6 & 0 & 0 & 92.6 & 0 & 0 \\
0 & 0 & 0 & 0 & 0 & 0 & 0 & 0 & 0 & 1 \\
1 & 0 & 0 & 0 & 0 & 0 & 0 & 0 & 0 & 0 \\
0 & 0 & 1 & 0 & 0 & 0 & 0 & 0 & 0 & 0 \\
0 & 0 & 0 & 0 & 0 & 1 & 0 & 0 & 0 & 0 \\
0 & 0 & 0 & 0 & 0 & 92.6 & 0 & 0 & 0 \\
0 & 0 & 0 & 0 & 0 & 0 & 1 & 0 & 0 & 0
\end{array}\right]
\end{aligned}
$$


$B_{u}=\left[\begin{array}{rrrrrrr}-0.0629 & 0.0629 & -0.1819 & 0.1819 & 0 & 0 & 0 \\ 0.0107 & 0.0107 & -0.0676 & -0.0676 & -0.1455 & -0.1455 & -0.1494 \\ -0.0142 & 0.0142 & -0.0128 & 0.0128 & 0 & 0 & 0 \\ 0 & 0 & 0 & 0 & 0 & 0 & 0 \\ 0 & 0 & -0.0098 & -0.0098 & -0.0071 & -0.0071 & -0.0074 \\ 0 & 0 & 0 & 0 & 0 & 0 & 0 \\ 0 & 0 & 0 & 0 & 0 & 0 & 0 \\ 0 & 0 & 0 & 0 & 0 & 0 & 0 \\ 0 & 0 & 0 & 0 & 0 & 0 & 0 \\ 0 & 0 & 0 & 0 & 0 & 0 & 0\end{array}\right.$

$\left.\begin{array}{rrrrrrrr}0 & 0 & 0.0652 & 0.0185 & 0.0034 & 0.0019 & -0.0019 & -0.0034 \\ -0.1494-1.2860 & 0 & 0 & 0.0013 & 0.0035 & 0.0035 & 0.0013 \\ 0 & 0 & -0.1272 & -0.0929 & 0.0195 & 0.0111 & -0.0111 & -0.0195 \\ 0 & -0.3122 & 0 & 0 & 0.1999 & 0.1999 & 0.1999 & 0.1999 \\ -0.0074-0.0676 & 0 & 0 & -0.0004 & -0.0004 & -0.0004 & -0.0004 \\ 0 & 0 & 0.0078 & 0.0066 & 0.0001 & 0.0001 & -0.0001 & -0.0001 \\ 0 & 0 & 0 & 0 & 0 & 0 & 0 & 0 \\ 0 & 0 & 0 & 0 & 0 & 0 & 0 & 0 \\ 0 & 0 & 0 & 0 & 0 & 0 & 0 & 0 \\ 0 & 0 & 0 & 0 & 0 & 0 & 0 & 0\end{array}\right]$

$D_{u}=\left[\begin{array}{rrrrrrrrrrrrrrr}0 & 0 & 0 & 0 & 0 & 0 & 0 & 0 & 0 & 0 & 0 & 0 & 0 & 0 & 0 \\ 0 & 0 & 0 & 0 & 0 & 0 & 0 & 0 & -0.3122 & 0 & 0 & 0.1999 & 0.1999 & 0.1999 & 0.1999 \\ 0 & 0 & 0 & 0 & 0 & 0 & 0 & 0 & 0 & 0 & 0 & 0 & 0 & 0 & 0 \\ 0 & 0 & 0 & 0 & 0 & 0 & 0 & 0 & 0 & 0 & 0 & 0 & 0 & 0 & 0 \\ 0 & 0 & 0 & 0 & 0 & 0 & 0 & 0 & 0 & 0 & 0 & 0 & 0 & 0 & 0 \\ 0 & 0 & 0 & 0 & 0 & 0 & 0 & 0 & 0 & 0 & 0 & 0 & 0 & 0 & 0 \\ 0 & 0 & 0 & 0 & 0 & 0 & 0 & 0 & 0 & 0 & 0 & 0 & 0 & 0 & 0 \\ 0 & 0 & 0 & 0 & 0 & 0 & 0 & 0 & 0 & 0 & 0 & 0 & 0 & 0 & 0 \\ 0 & 0 & 0 & 0 & 0 & 0 & 0 & 0 & 0 & 0 & 0 & 0 & 0 & 0 & 0 \\ 0 & 0 & 0 & 0 & 0 & 0 & 0 & 0 & 0 & 0 & 0 & 0 & 0 & 0 & 0 \\ 0 & 0 & 0 & 0 & 0 & 0 & 0 & 0 & 0 & 0 & 0 & 0 & 0 & 0 & 0\end{array}\right]$




\section{Index}

fault detectability, 3

strong, 3

fault detection, 1

computational methods, 8

nullspace method, 4

residual generator, 3

fault detection and isolation problem, 5

strong isolation, 5

weak isolation, 5

fault detection problem, 3

fault monitoring, 9

component level, 10

system level, 12

fault signature matrix, 4 